ISSN 0103-8478

\title{
Análise qualitativa de compostos voláteis do headspace de carne cozida de ovinos e caprinos
}

\section{Qualitative analysis of headspace volatile compounds of lambs and goats cooked meat}

\author{
Jossiê Zamperetti Donadel ${ }^{\mathrm{I}}$ José Laerte NörnbergI Julcemar Dias Kessler ${ }^{\mathrm{II}}$

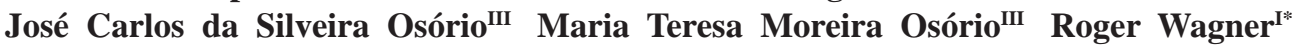

RESUMO

Este estudo teve como objetivo caracterizar qualitativamente os compostos voláteis (CV) do headspace de diferentes músculos de carne cozida de ovinos e caprinos da região do Alto Camaquã, Rio Grande do Sul - Brasil. Os CV do headspace dos músculos cozidos, L. dorsi e V. lateralis de cinco ovinos machos (idade de 6 meses) e cinco cabritos machos castrados (idade de 8-9 meses) foram analisados pela técnica de microextração em fase sólida (HS-SPME) e cromatógrafo a gás acoplado a espectrômetro de massas (GC/MS). Foram encontrados 73 compostos voláteis, dentre eles aldeídos, cetonas e compostos sulfurados, característicos de carne processada termicamente. A partir da fração volátil, foi possivel encontrar marcadores que discriminassem as espécies animais estudadas, caracterizando os caprinos por apresentarem, entre outros compostos, terpenos ( $\beta$-pineno, $\alpha$-gurjuneno, $\alpha$-muuroleno), ausentes em ovinos. Alguns compostos discriminaram $\mathbf{V}$. lateralis $e \mathbf{L}$. dorsi de ovinos, como álcool isopropílico, $\alpha$-pineno, o-xileno, porém não foi possivel obter uma diferenciação entre os músculos de caprinos.

Palavras-chave: marcador de qualidade, cromatografia em fase gasosa, músculos, microextração em fase sólida.

\section{ABSTRACT}

The aim of this research was to characterize qualitatively the potential markers volatile compounds of the headspace of cooked lambs and goats meat from region of Alto Camaquã, Rio Grande do Sul-Brazil. The volatile compounds of cooked meat of $\mathbf{L}$. dorsi and $\mathbf{V}$. lateralis muscles of five male lambs (6 months of age) and five castrated male goats ( $8-9$ months of age) were analyzed by solid phase microextraction (HS-SPME) technic and gas chromatography coupled to mass spectrometer $(G C / M S)$. Seventy-three volatile compounds were found in samples headspace, including aldehydes, ketones and sulfur compounds characteristic from processed meat. Furthermore, it was possible to discriminate goats meat mainly by identification of terpenes ( $\beta$-pinene, $\alpha$-gurjunene, $\alpha$-muurolene), which were absent in lambs. Some compounds discriminated $\mathbf{V}$. lateralis and $\mathbf{L}$. dorsi lamb muscles, such as isopropyl alcohol, $\alpha$-pinene, m-xylene, but there was no discrimination between the muscles of goats.

Key words: muscles, HS-SPME, gas chromatography, quality marker.

\section{INTRODUÇÃO}

Carnes de diferentes animais e cortes podem ser discriminadas pelos consumidores através de parâmetros sensoriais combinados de aparência, textura e aroma/sabor. Nesse sentido, uma forte contribuição na qualificação da carne pode ser atribuída à presença de diferentes substâncias voláteis, que conferem sabor e aroma (MADRUGA, 1997; MOTTRAM, 1998; VASTA \& PRIOLO, 2006).

Vários fatores podem afetar diretamente a composição da matéria-prima, como a influência da raça (ELMORE et al., 2000a), da dieta (VASTA \& PRIOLO, 2006), da castração e idade de abate (SUTHERLAND \& AMES, 1995), entre outros, e, consequentemente, na qualidade da carne. Além da matéria-prima, os parâmetros do processamento também estão relacionados com o desenvolvimento do

'Departamento de Tecnologia e Ciência dos Alimentos, Centro de Ciências Rurais (CCR), Universidade Federal de Santa Maria (UFSM), Av. Roraima, 1000, Prédio 42, Santa Maria, RS, Brasil. E-mail: rogerwag@gmail.com. *Autor para correspondência.

"Departamento de Zootecnia, Centro de Educação Superior do Oeste (CEO),Universidade do Estado de Santa Catarina (UDESC), Chapecó, SC, Brasil.

IIIPós-graduação em Zootecnia, Universidade Federal da Grande Dourados (UFGD), Dourados, MS, Brasil. 
sabor de carne processada termicamente. Esse sabor característico é proveniente de reações químicas, como reação de Maillard, oxidação lipídica e proteica (MOTTRAM, 1998), envolvendo diferentes precursores (MADRUGA, 1994; MADRUGA, 1997). Muitos dos compostos voláteis oriundos dessas reações são responsáveis pelo odor e sabor de carne processada termicamente. A caracterização dos CV envolve basicamente o isolamento destes da matriz, por meio de técnicas de extração, seguido da análise quali e quantitativa em cromatógrafo a gás acoplado a espectrômetro de massas (GC/MS).

As carnes com características definidas fornecem padrões de reconhecimento que podem ser associadas à denominação de origem, e, consequentemente, agregar valor ao produto de uma determinada região geográfica. Assim, a determinação dos compostos voláteis apresenta potencial de levantamento de critério de qualidade diretamente para o odor e sabor da carne cozida. Desse modo, o objetivo do presente trabalho foi caracterizar qualitativamente os compostos voláteis, presentes no headspace de carne cozida de diferentes músculos (Vastus lateralis e Longissimus dorsi) de ovinos e caprinos para identificar compostos potenciais marcadores de qualidade.

\section{MATERIAL E MÉTODOS}

\section{Amostras}

A pesquisa foi conduzida na cidade de Bagé, Rio Grande Sul - Brasil, localizada dentro da área geográfica do Território do Alto Camaquã. O sistema de criação desenvolvido foi o extensivo com ovinos da raça Corriedale e caprinos sem raça definida (SRD). A alimentação foi exclusivamente de pastagem nativa e do estrato arbóreo-arbustivo da região. Foram utilizadas 20 amostras, sendo de ovinos machos (5 músculos de $\boldsymbol{L}$. dorsi e 5 de $\boldsymbol{V}$. lateralis) com idade de 6 meses e de cabritos machos castrados (5 músculos de L. dorsi e 5 de V. lateralis), com idade de 8-9 meses. As carcaças dos animais abatidos foram acondicionadas em uma câmara fria com temperatura de $1^{\circ} \mathrm{C}$ por um período de $18 \mathrm{~h}$, para que ocorresse o processo de modificações bioquímicas (rigor mortis), necessárias para a transformação de músculo em carne. Após, foram retirados os músculos Longissimus dorsi (lombo), Vastus lateralis (perna), para determinação dos compostos voláteis. As amostras foram embaladas em sacos plásticos, identificadas e armazenadas em freezer a $-18^{\circ} \mathrm{C}$, sendo mantidas nessa temperatura até a realização das análises.
Preparo das amostras

As amostras, antes das análises, foram submetidas a um tratamento térmico adaptado de ELMORE et al. (2000b) para a caracterização dos voláteis. Elas foram descongeladas em temperatura de $5^{\circ} \mathrm{C}$ por $12 \mathrm{~h}$ e cortadas em pedaços com $30 \pm 0,1 \mathrm{~g}$, os quais foram fatiados em duas partes iguais, submetidos ao cozimento em autoclave, à temperatura de $121^{\circ} \mathrm{C}$, durante $30 \mathrm{~min}$. Posteriormente, as amostras foram resfriadas à temperatura ambiente, trituradas em processador de alimentos. Em seguida, $5 \mathrm{~g}$ de amostra foram pesados em um frasco de $20 \mathrm{~mL}$ e submetidos à extração de $\mathrm{CV}$.

\section{Microextração em fase sólida (HS-SPME)}

Para o isolamento dos CV das matrizes, foi utilizada a técnica de microextração em fase sólida no espaço confinado (headspace) entre a amostra e o frasco vedado. A fibra de revestimento Car/ PDMS (carboxen/polidimetilsiloxano, $75 \mu \mathrm{m} \times 10 \mathrm{~mm}$ Supelco, Bellefonte, PA, USA) foi exposta ao headspace da amostra por $45 \mathrm{~min}$, em banho-maria a $60^{\circ} \mathrm{C}$. Antes da extração, cada frasco contendo as amostras foi submetido à mesma temperatura de extração, sem exposição da fibra por 10min (tempo de equilíbrio). Após o término da extração, a fibra foi removida e inserida no injetor do cromatógrafo para dessorção térmica dos analitos.

\section{Análises cromatográficas}

Os compostos voláteis foram separados e identificados em cromatógrafo a gás, acoplado a um espectrômetro de massas (Shimadzu GC/MS-QP 2010 Plus). A dessorção térmica dos analitos da fibra ocorreu no injetor do $\mathrm{GC}$ a $250^{\circ} \mathrm{C}$, no modo splitless, utilizando um insersor de $0,75 \mathrm{~mm}$ de diâmetro interno, sendo que a fibra foi mantida exposta no interior do injetor por 10min, com o objetivo de eliminar o efeito memória. Os $\mathrm{CV}$ foram separados em uma coluna capilar de fase polar de polietilenoglicol (PEG), Chrompack WAX 52-CB $(60 \mathrm{~m} \times 0,25 \mathrm{mmd} . \mathrm{i} \times 25 \mu \mathrm{m})$. $\mathrm{O}$ programa de temperatura da coluna iniciou em $35^{\circ} \mathrm{C}$, permanecendo por 2 minutos, posteriormente, aumentando até $80^{\circ} \mathrm{C}$ a uma taxa de $2^{\circ} \mathrm{C} \min ^{-1}$. A partir dessa temperatura, aumentou-se até $150^{\circ} \mathrm{C}$ com uma rampa de aquecimento de $4^{\circ} \mathrm{C} \min ^{-1}$, então até $230^{\circ} \mathrm{C}$ com taxa de $8^{\circ} \mathrm{C} \mathrm{min}^{-1}$, permanecendo em isoterma por 5 minutos. $\mathrm{O}$ gás de arraste utilizado foi o He, com pressão constante e uma vazão inicial de $2 \mathrm{~mL} \mathrm{~min}^{-1}$. O analisador de massas quadrupolo foi operado no modo de varredura, monitorando as massas de 35 a $350 \mathrm{~m} / \mathrm{z}$. Nos experimentos, as amostras foram comparadas pelas áreas geradas da 
integração dos picos cromatográficos. Os compostos foram identificados, primeiramente, por comparação de seus espectros de massa, com os do banco de espectros da biblioteca do GC/MS (NIST 2.0), bem como com índice de retenção experimental com o da literatura (ACREE \& HEINRINCH 2012; ElSAYED, 2012).

\section{Análise estatística}

Os dados obtidos foram analisados aplicando uma análise exploratória dos dados através da Análise de Componentes Principais (PCA), com o objetivo de visualizar a correlação entre as variáveis e os agrupamentos entre as amostras, empregando o programa estatístico Pirouette 3.11 .

\section{RESULTADOS E DISCUSSÃO}

\section{Compostos voláteis}

Na análise do headspace, para os músculos

L. dorsi e V. lateralis de carnes de ovinos e caprinos, foram detectados 73 compostos voláteis, dos quais 72 foram identificados como sendo pertencentes às classes químicas: ácido, hidrocarboneto, álcool, aldeído, cetona, éster, furano e terpeno, bem como compostos sulfurados e nitrogenados. As tabelas $1 \mathrm{e}$ 2 apresentam os compostos voláteis identificados nas amostras estudadas.

Aldeídos de cadeia linear saturados de 4 a 9 átomos de carbono foram identificados para os dois músculos de ambas as espécies, exceto octanal em L. dorsi de caprinos, sendo detectados em maior abundância na carne ovina. Esses compostos normalmente são derivados da autoxidação de ácidos graxos, presentes nos triglicerídeos intramusculares e fosfolipídios estruturais (MADRUGA et al., 2009). Benzaldeído foi encontrado em ambos os animais, sendo que VASTA et al. (2011) reportaram a presença desse composto em L. dorsi de carne bovina, indicando sua origem a partir da degradação do ácido linolênico (18:3 n3), que é o principal ácido graxo presente em plantas herbáceas verdes (ELMORE et al., 2005) e, de acordo com SONDERGAARD \& STAHNKE (2002), esse analito pode provir também da degradação de aminoácidos aromáticos.

Ácidos e alcoois de cadeia linear formada de 5 a 9 átomos de carbono e cetonas foram encontrados no headspace das amostras e esses compostos têm sido reportados na literatura como oriundos da oxidação lipídica (DRANSFIELD, 2008; MOTTRAM, 1998). Os ácidos butanoico, pentanoico, hexanoico, heptanoico e octanoico foram encontrados no headspace para os dois músculos de ambas as espécies, sendo majoritários para $\mathbf{L}$. dorsi de ovinos. Esses analitos são marcadores de qualidade importantes da carne e devem estar presentes em baixa concentração, pois estão correlacionados com aroma de ranço (SONDERGAARD \& STAHNKE, 2002). Hexanol e 1-octanol foram encontrados nos dois tipos de músculos, obtendo-se maior quantidade em ovinos. As principais cetonas identificadas foram 2-propanona, 2-butanona, 6-metil-5hepten-2-ona, 2,3-pentanodiona, sendo que os três primeiros compostos foram encontrados em maiores proporções em relação aos ovinos. 2,3-octanodiona foi detectada para todos os músculos de ambas as espécies, sendo reportada em outro estudo como um importante marcador de alimentação a base de pastagem (VASTA \& PRIOLO, 2006). 2-octanona foi encontrada somente no headspace para os dois músculos de ovinos, portanto um potencial marcador para discriminar os animais.

Os compostos dimetil di e trissulfeto foram encontrados em ambas as espécies, além de dimetil sulfona. Em outro estudo, esses sulfetos foram relacionados com o aroma de carne cozida e grelhada e são provenientes da degradação de aminoácidos sulfurados, como cisteína e metionina (MOTTRAM, 1998). Além disso, YOUNG et al. (2003) reportaram que dimetil sulfeto e dimetil sulfona isolados de gordura de cordeiro cozida foram positivamente correlacionados com atividade de pastejo. A presença de 3-metiltiofeno em proporções consideráveis no headspace de todas as amostras de carne pode indicar a ocorrência de desfosforilação e desidratação de fosfato-ribose, a qual forma tiofenos a partir de furanos (MOTTRAM, 1998).

Analitos identificados neste experimento como 2 e 3-metilbutanal, 2-metil-, 2-etil- e 2-pentilfurano, furfural foram também reportados em estudos realizados por MADRUGA et al. (2009) em carne caprina. Os autores sugerem que esses compostos surgem a partir da degradação de Strecker da reação de Maillard, proveniente do cozimento. Nessa etapa, ocorrem reações complexas entre os componentes não voláteis dos tecidos magro e gorduroso para originar $\mathrm{CV}$, como a proteólise e degradação dos aminoácidos leucina e isoleucina (ESTÉVEZ, 2011). Esses compostos contribuem para a sensação do aroma característico de carne processada termicamente (MOTTRAM, 1998).

A presença de 2-acetiltiazol é um marcador importante de carne cozida. Possivelmente, sua formação envolve a reação de compostos dicarbonílicos ou hidroxicetonas com sulfeto de hidrogênio e amônia, formada através da hidrólise 
Tabela 1 - Compostos voláteis de carne cozida de ovinos e caprinos dos músculos V. lateralis e L. dorsi.

\begin{tabular}{|c|c|c|c|c|c|c|c|c|c|c|}
\hline \multirow{3}{*}{ Composto } & \multirow{3}{*}{$\mathrm{IK}^{\mathrm{a}}$} & \multirow{3}{*}{$\mathrm{I}^{\mathrm{b}}$} & \multicolumn{4}{|c|}{ 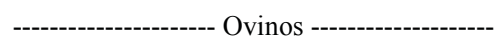 } & \multicolumn{4}{|c|}{ 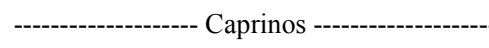 } \\
\hline & & & \multicolumn{2}{|c|}{--- V. Lateralis ---- } & \multicolumn{2}{|c|}{---- L. Dorsi ----- } & \multicolumn{2}{|c|}{---- V. Lateralis ---- } & \multicolumn{2}{|c|}{---- L. Dorsi ---- } \\
\hline & & & $\mathrm{A}^{\mathrm{c}}$ & $\mathrm{DP}^{\mathrm{d}}$ & A & DP & A & DP & A & DP \\
\hline 2-metilpropanal & 817 & $\mathrm{a}$ & 6,45 & 2,31 & 11,04 & 0,58 & 1,20 & 1,77 & 2,83 & 1,57 \\
\hline 2-propanona & 824 & $\mathrm{a}$ & 45,54 & 13,36 & 54,72 & 1,35 & 22,36 & 5,96 & 27,51 & 4,66 \\
\hline 2-octeno & 856 & $\mathrm{a}$ & 17,03 & 6,48 & 15,46 & 0,23 & - & - & - & - \\
\hline 2-metilfurano & 875 & $\mathrm{a}$ & 19,57 & 4,68 & 14,02 & 0,51 & 14,74 & 3,79 & 10,74 & 2,59 \\
\hline butanal & 883 & $\mathrm{a}$ & 17,83 & 3,71 & 11,03 & 0,39 & 8,50 & 3,40 & 7,00 & 2,96 \\
\hline acetato de etila & 892 & $\mathrm{a}$ & 13,86 & 7,60 & 10,45 & 0,56 & 14,65 & 5,52 & 5,48 & 1,26 \\
\hline acetato de vinila & 902 & $\mathrm{~b}$ & 6,91 & 3,96 & 19,12 & 0,85 & - & - & 3,91 & 1,02 \\
\hline 2-butanona & 910 & $\mathrm{a}$ & 46,73 & 20,55 & 45,41 & 0,93 & 16,05 & 10,49 & 19,95 & 7,79 \\
\hline 2-metilbutanal & 919 & $\mathrm{a}$ & 0,67 & 0,27 & 5,03 & 0,08 & 0,36 & 0,38 & 0,69 & 0,39 \\
\hline 3-metilbutanal & 923 & $\mathrm{a}$ & 2,09 & 0,98 & 10,38 & 0,18 & 0,78 & 0,88 & 1,61 & 0,54 \\
\hline álcool isopropílico & 936 & $\mathrm{a}$ & 1,95 & 1,04 & - & - & - & - & - & - \\
\hline 2-etilfurano & 958 & $\mathrm{~b}$ & 3,21 & 3,09 & 13,66 & 0,65 & 1,42 & 0,80 & 2,77 & 1,08 \\
\hline pentanal & 986 & $\mathrm{a}$ & 14,03 & 11,49 & 10,99 & 0,20 & 6,65 & 2,92 & 6,32 & 4,36 \\
\hline butanoato de metila & 997 & $\mathrm{a}$ & 2,60 & 0,48 & 1,97 & 0,07 & 1,56 & 0,53 & 0,71 & 0,10 \\
\hline$\alpha$-pineno & 1018 & $\mathrm{a}$ & 1,19 & 0,91 & - & - & 2,82 & 3,56 & 4,38 & 1,97 \\
\hline 2,3-pentanodiona & 1069 & $\mathrm{a}$ & 1,95 & 1,18 & 3,11 & 0,11 & 2,14 & 1,54 & 2,26 & 0,80 \\
\hline dimetil dissulfeto & 1077 & $\mathrm{a}$ & 2,33 & 2,68 & 1,63 & 0,06 & 1,32 & 0,73 & 0,50 & 0,00 \\
\hline acetato de butila & 1080 & $\mathrm{a}$ & 0,81 & 0,18 & 1,62 & 0,22 & - & - & - & - \\
\hline hexanal & 1089 & $\mathrm{a}$ & 107,49 & 71,09 & 57,99 & 1,85 & 65,43 & 41,54 & 62,21 & 36,15 \\
\hline 3-metiltiofeno & 1095 & $\mathrm{a}$ & 67,69 & 41,23 & 27,68 & 0,52 & 47,02 & 23,06 & 31,68 & 14,33 \\
\hline$\beta$-pineno & 1098 & $\mathrm{a}$ & - & - & - & - & 18,84 & 0,00 & 10,71 & 8,66 \\
\hline etilbenzeno & 1124 & $\mathrm{a}$ & 5,10 & 3,13 & 8,20 & 0,39 & 6,74 & 3,74 & 4,52 & 2,85 \\
\hline p-xileno & 1138 & $\mathrm{a}$ & 0,92 & 0,49 & 1,17 & 0,11 & - & - & - & - \\
\hline m-xileno & 1147 & $\mathrm{a}$ & - & - & 1,33 & 0,04 & 1,46 & 1,21 & 1,02 & 0,00 \\
\hline butanol & 1168 & $\mathrm{a}$ & 0,98 & 0,00 & 0,96 & 0,00 & 1,11 & 0,54 & 0,37 & 0,12 \\
\hline heptanal & 1186 & $\mathrm{a}$ & 50,71 & 43,07 & 71,04 & 5,64 & 14,43 & 15,70 & 7,00 & 5,11 \\
\hline 2-pentilfurano & 1222 & $\mathrm{a}$ & 7,77 & 6,26 & 34,15 & 1,29 & 1,89 & 0,78 & 1,96 & 0,56 \\
\hline hexanoato de etila & 1233 & $\mathrm{a}$ & 0,76 & 0,09 & 0,43 & 0,00 & - & - & - & - \\
\hline 1-propoxi-2-propanol & 1251 & $\mathrm{~b}$ & 1,91 & 0,67 & 0,44 & 0,00 & 1,48 & 0,98 & 1,02 & 0,56 \\
\hline estireno & 1258 & $\mathrm{a}$ & 39,40 & 29,57 & 81,13 & 3,33 & 43,00 & 21,92 & 6,77 & 4,36 \\
\hline p-cimeno & 1265 & $\mathrm{a}$ & - & - & - & - & 1,69 & 0,84 & 2,09 & 0,92 \\
\hline 2-octanona & 1285 & $\mathrm{a}$ & 0,63 & 0,18 & 1,27 & 0,01 & - & - & - & - \\
\hline octanal & 1286 & $\mathrm{a}$ & 11,66 & 6,59 & 21,97 & 1,98 & 13,74 & 11,43 & - & - \\
\hline acetoína & 1296 & $\mathrm{a}$ & 4,40 & 3,13 & 8,72 & 0,55 & 5,12 & 4,41 & - & - \\
\hline acetol & 1313 & $\mathrm{a}$ & 12,02 & 7,15 & 33,04 & 1,38 & 3,44 & 0,41 & 2,42 & 0,92 \\
\hline 2-hexilfurano & 1326 & $\mathrm{a}$ & - & - & 5,32 & 0,56 & - & - & - & - \\
\hline 2,3-octanodiona & 1330 & $\mathrm{a}$ & 9,92 & 2,84 & 5,98 & 0,12 & 0,39 & 0,24 & 4,23 & 3,41 \\
\hline
\end{tabular}

a Índice de Kovats experimental em coluna capilar Chrompack WAX 52-CB, Varian Chrompack; ${ }^{\mathrm{b}}$ Confiabilidade da identificação - a espectro de massas e índice de Kovats em concordância com os dados da literatura; b - espectro de massas de acordo com a biblioteca NIST 02 (tentativamente identificado). ${ }^{\mathrm{c}}$ área média $\times 10^{5}(\mathrm{n}=5) .{ }^{\mathrm{d}}$ Desvio padrão das áreas.

da cisteína e aldeídos ou pela degradação de Strecker (MOTTRAM, 1998).

Nas amostras, foram encontrados terpenos, como eudesmano, copaeno, cariofileno, $\alpha$-pineno, comuns entre as duas espécies. Já outros terpenos como p-cimeno, $\alpha$-gurjuneno, $\beta$-pineno, $\alpha$-muuroleno e calamaneno estavam presentes somente no headspace de V. lateralis e $\mathbf{L}$. dorsi de caprinos. A ocorrência desses compostos pode ser atribuída a uma alimentação baseada em uma dieta herbácea, já que os animais estudados alimentavam-se de pasto nativo e estrato arbóreo-arbustivo da região. Moléculas terpenoides representam uma pequena porcentagem de todos os compostos voláteis de carne de ruminantes. Terpenos 
Tabela 2 - Compostos voláteis de carne cozida de ovinos e caprinos dos músculos V. lateralis e L. dorsi.

\begin{tabular}{|c|c|c|c|c|c|c|c|c|c|c|}
\hline \multirow{3}{*}{ Composto } & \multirow{3}{*}{$\mathrm{IK}^{\mathrm{a}}$} & \multirow{3}{*}{$\mathrm{I}^{\mathrm{b}}$} & \multicolumn{4}{|c|}{------------------- Ovinos ----------------' } & \multicolumn{4}{|c|}{----------------- Caprinos ----------------- } \\
\hline & & & \multicolumn{2}{|c|}{--- V. Lateralis --- } & \multicolumn{2}{|c|}{---- L. Dorsi ----- } & \multicolumn{2}{|c|}{--- V. Lateralis --- } & \multicolumn{2}{|c|}{---- L. Dorsi ---- } \\
\hline & & & $A^{c}$ & $\mathrm{DP}^{\mathrm{d}}$ & A & DP & A & DP & A & DP \\
\hline 6-metil-5-hepten-2-ona & 1343 & $\mathrm{a}$ & 2,59 & 0,98 & 4,43 & 0,17 & 1,44 & 0,02 & 1,22 & 0,49 \\
\hline hexanol & 1361 & $\mathrm{a}$ & 5,03 & 3,02 & 6,77 & 0,16 & 2,58 & 1,48 & 2,50 & 0,68 \\
\hline dimetil trissulfeto & 1372 & $\mathrm{a}$ & 4,41 & 1,82 & 3,93 & 0,04 & 1,19 & 0,80 & 0,85 & 0,26 \\
\hline nonanal & 1393 & $\mathrm{a}$ & 28,86 & 9,19 & 35,66 & 1,27 & 21,88 & 9,60 & 10,69 & 2,12 \\
\hline 1-octen-3-ol & 1454 & $\mathrm{a}$ & 7,58 & 3,21 & 10,19 & 0,17 & 6,71 & 3,12 & 4,90 & 1,32 \\
\hline ácido acético & 1459 & $\mathrm{a}$ & 18,39 & 10,55 & 32,76 & 1,39 & 10,76 & 2,93 & 12,48 & 5,26 \\
\hline furfural & 1477 & $\mathrm{a}$ & 2,65 & 0,76 & 6,90 & 0,08 & 0,45 & 0,11 & 0,53 & 0,21 \\
\hline diacetato de 1,2-etanediol & 1481 & $\mathrm{a}$ & 15,57 & 2,20 & 15,88 & 0,29 & - & - & - & - \\
\hline copaeno & 1484 & $\mathrm{a}$ & 1,88 & 0,00 & 0,32 & 0,00 & 6,80 & 3,46 & 4,90 & 3,06 \\
\hline 2-etilhexanol & 1491 & $\mathrm{a}$ & 3,21 & 2,87 & 6,29 & 0,27 & - & - & - & - \\
\hline$\alpha$-gurjuneno & 1523 & $\mathrm{a}$ & - & - & - & - & 11,15 & 4,70 & 8,60 & 3,65 \\
\hline benzaldeído & 1533 & $\mathrm{a}$ & 53,12 & 13,27 & 84,64 & 1,55 & 16,38 & 3,82 & 19,53 & 5,39 \\
\hline ácido propanoico & 1547 & $\mathrm{a}$ & 1,48 & 0,99 & 2,56 & 0,12 & 1,11 & 0,42 & 1,45 & 0,51 \\
\hline 1-octanol & 1563 & $\mathrm{a}$ & 1,97 & 0,46 & 2,83 & 0,09 & 1,78 & 0,82 & 1,05 & 0,17 \\
\hline 2,3-butanediol & 1588 & $\mathrm{a}$ & - & - & - & - & 2,58 & 2,44 & 0,72 & 0,02 \\
\hline eudesmano & 1588 & $\mathrm{~b}$ & 2,58 & 0,37 & 2,39 & 0,00 & 5,42 & 2,01 & 2,75 & 1,21 \\
\hline$\beta$-cariofileno & 1591 & $\mathrm{a}$ & 2,76 & 1,59 & 1,72 & 0,12 & 18,00 & 6,71 & 14,29 & 6,81 \\
\hline (E)2-octen-1-ol & 1622 & $\mathrm{a}$ & 0,41 & 0,12 & 0,81 & 0,02 & - & - & - & - \\
\hline ácido butanoico & 1633 & $\mathrm{a}$ & 29,22 & 16,33 & 43,77 & 1,88 & 13,09 & 2,69 & 12,10 & 3,57 \\
\hline butirolactona & 1653 & $\mathrm{a}$ & 24,68 & 10,61 & 22,33 & 0,94 & 12,73 & 3,92 & 9,56 & 2,94 \\
\hline 2-acetiltiazol & 1663 & $\mathrm{a}$ & 1,03 & 0,23 & 2,56 & 0,04 & 1,11 & 0,45 & 1,46 & 0,60 \\
\hline álcool furfurílico & 1674 & $\mathrm{a}$ & 0,96 & 0,40 & 3,64 & 0,10 & - & - & - & - \\
\hline $\mathrm{ni}^{\mathrm{e}}$ & 1677 & -- & - & - & - & - & 22,23 & 8,61 & 17,85 & 2,74 \\
\hline 2-formiltiofeno & 1714 & $\mathrm{~b}$ & 0,94 & 0,44 & 0,79 & 0,03 & 0,45 & 0,13 & - & - \\
\hline$\alpha$-muuroleno & 1732 & $\mathrm{a}$ & - & - & - & - & 2,23 & 0,21 & 1,25 & 0,70 \\
\hline ácido pentanoico & 1745 & $\mathrm{a}$ & 0,74 & 0,25 & 1,40 & 0,03 & 0,60 & 0,15 & 0,65 & 0,18 \\
\hline calameneno & 1845 & $\mathrm{a}$ & - & - & - & - & 2,58 & 1,27 & 2,10 & 0,71 \\
\hline ácido hexanoico & 1851 & $\mathrm{a}$ & 11,28 & 5,68 & 15,52 & 0,40 & 6,09 & 0,88 & 4,84 & 0,92 \\
\hline calacoreno & 1934 & $\mathrm{a}$ & - & - & - & - & 0,74 & 0,10 & 0,52 & 0,26 \\
\hline dimetil sulfona & 1940 & $\mathrm{a}$ & 0,77 & 0,47 & 0,64 & 0,01 & 0,75 & 0,42 & 1,18 & 0,65 \\
\hline ácido heptanoico & 1958 & $\mathrm{a}$ & 0,96 & 0,19 & 1,77 & 0,03 & 0,49 & 0,03 & 0,46 & 0,15 \\
\hline 2-acetilpirrol & 1997 & $\mathrm{~b}$ & - & - & 2,25 & 0,08 & - & - & - & - \\
\hline$\delta$-octalactona & 2009 & $\mathrm{a}$ & 0,78 & 0,26 & 0,59 & 0,02 & - & - & - & - \\
\hline fenol & 2022 & $\mathrm{a}$ & 0,87 & 0,22 & 0,57 & 0,04 & - & - & - & - \\
\hline furaneol & 2054 & $\mathrm{a}$ & 0,93 & 0,15 & 1,31 & 0,05 & - & - & - & - \\
\hline ácido octanoico & 2065 & $\mathrm{a}$ & 5,17 & 1,60 & 6,31 & 0,05 & 3,88 & 0,54 & 3,23 & 0,09 \\
\hline
\end{tabular}

a Índice de Kovats experimental em coluna capilar Chrompack WAX 52-CB, Varian Chrompack; ${ }^{\mathrm{b}}$ Confiabilidade da identificação - a espectro de massas e índice de Kovats em concordância com os dados da literatura; b - espectro de massas de acordo com a biblioteca NIST 02 (tentativamente identificado). ${ }^{\mathrm{c}}$ área média $\times 10^{5}(\mathrm{n}=5) .{ }^{\mathrm{d}}$ Desvio padrão das áreas. ${ }^{\mathrm{e}}$ composto não identificado.

são sintetizados quase que exclusivamente no reino vegetal e a sua presença na carne de ruminantes ou em produtos lácteos pode ser considerada como um indicador de dietas herbáceas (VASTA \& PRIOLO, 2006). PRIOLO et al. (2004) reportaram a presença de $\beta$-cariofileno em gordura perirrenal de cordeiro. Estudos realizados por MARIACA et al. (1997) e PRIOLO et al. (2004) propuseram o $\beta$-cariofileno como um biomarcador de um sistema de alimentação com pasto. CORNU et al. (2001) demonstraram que esse sesquiterpeno foi um dos componentes principais entre todos os terpenos de uma amostra de plantas naturais de pastagem. Assim, os terpenos encontrados na carne podem ser considerados como um marcador importante na discriminação entre espécies, podendose inferir que caprinos se alimentavam de espécies de 
plantas diferentes de ovinos, já que estavam localizados no mesmo sistema de criação extensivo. Além disso, alguns compostos como álcool isopropílico e $\alpha$-pineno diferenciaram os músculos de ovinos, encontrando-se somente no V. lateralis, enquanto que 2-acetilpirrol foi detectado somente em $\boldsymbol{L}$. dorsi. O composto fenol foi encontrado somente nos músculos de ovinos. A presença de fenóis livres pode surgir de uma transferência direta de forragem verde para a carne ou pode ser sintetizado por micro-organismos do rúmen (HA \& LINDSAY, 1991), através da quebra da lignina em fenóis monoméricos, conforme CHEN et al. (1985).

O composto $\delta$-octalactona foi encontrada somente em headspace de ovinos, enquanto que butirolactona foi detectada com maior abundância em V. lateralis, para ambas as espécies, obtendo-se maiores concentrações em ovinos. Lactonas derivam de seus hidróxi-ácidos graxos (GARGOURI et al., 2003), os quais são formados no rúmen pela oxidação dos ácidos oleico e linoleico da dieta (URBACH 1990).

\section{Análise estatística}

A Análise de Componentes Principais foi aplicada aos dados como ferramenta exploratória, reunindo as informações redundantes em um sistema bidimensional. Assim, o conjunto de dados foi composto por 19 amostras de carne de caprinos e ovinos de ambos os músculos e foram selecionados 65 compostos voláteis (variáveis) dos 73 analitos. Uma amostra de $\boldsymbol{L}$. dorsi de caprinos apresentou um cromatograma anômalo e foi retirada da matriz de dados para a PCA.

A figura 1 apresenta os resultados da PCA através dos gráficos das amostras e das variáveis. Os dois

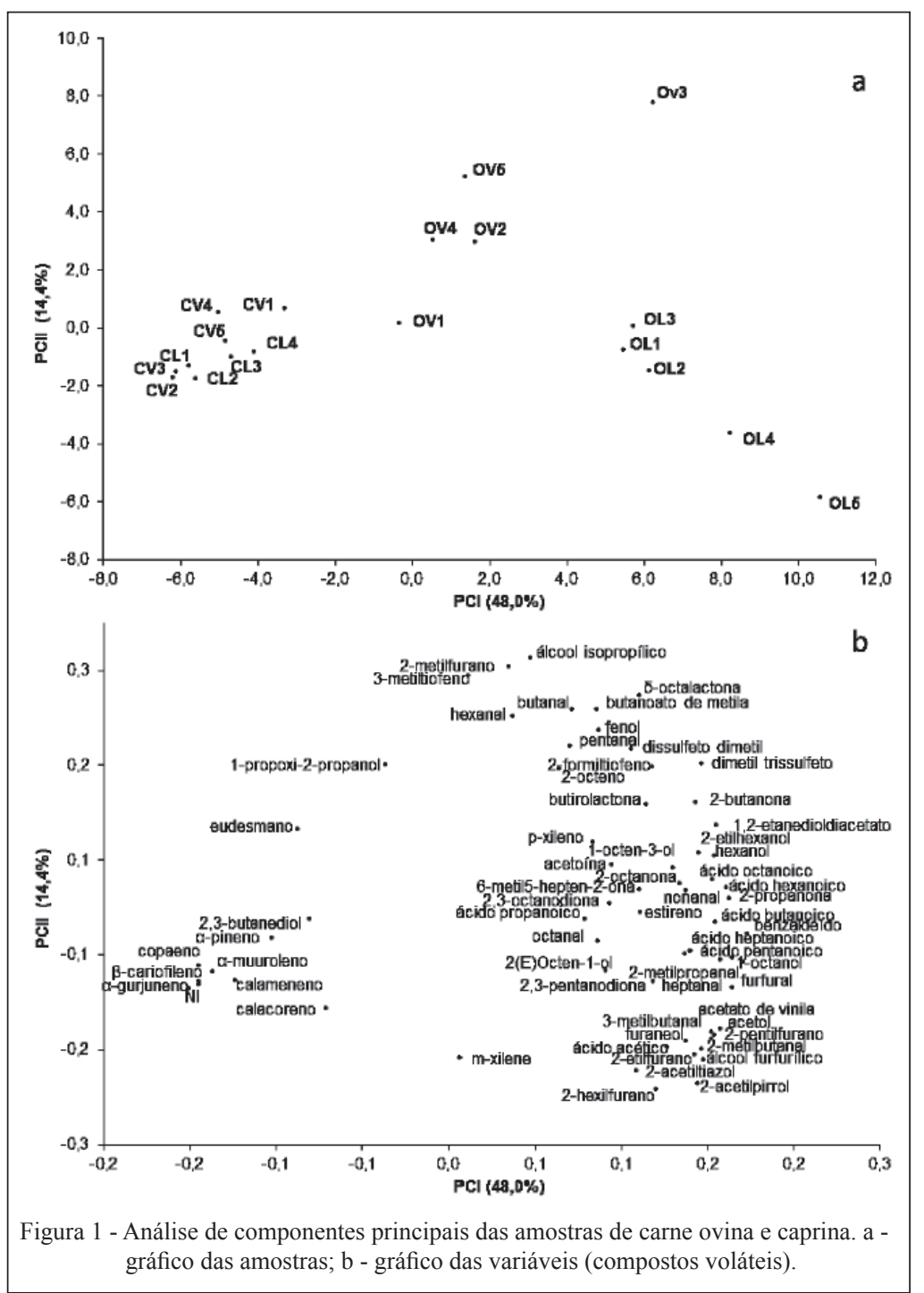

Ciência Rural, v.43, n.11, nov, 2013. 
primeiros componentes principais somaram $62,4 \%$ (PC I e PC II) da variância dos dados. O PC I, com 48,0\% da variância explicada, discriminou as espécies animais, sendo que os ovinos apresentaram, de uma forma geral, elevada quantidade de compostos, produto da oxidação lipídica, como butanal, hexanal, heptanal, além dos ácidos butanoico, hexanoico, heptanoico e octanoico e cetonas como 2-propanona e 2-butanona. Por outro lado, os caprinos foram correlacionados com abundância dos terpenos calamaneno, $\alpha$-gurjuneno, copaeno, cariofileno, eudesmano e $\alpha$-muuroleno. O PC II $(14,4 \%)$ permitiu distinguir os tratamentos de carne ovina, separandoas pelo tipo de músculo. No headspace das carnes provenientes do $\boldsymbol{L}$. dorsi de ovinos, foram encontrados os compostos 2-hexilfurano, m-xileno, 2-acetilpirrol com exclusividade e os compostos 2-pentilfurano, 2,3-pentanodiona, furfural, 2- e 3-metilbutanal, furaneol, acetol em maior abundância. Entretanto, o $\mathbf{V}$. lateralis apresentou maior abundância dos compostos 2-octeno, 2-metilfurano, pentanal, dissulfeto dimetil, 3-metiltiofeno. Não foi possível encontrar compostos que discriminassem de forma efetiva entre os músculos das carnes caprinas.

\section{CONCLUSÃO}

Neste trabalho, foi possível identificar os compostos voláteis encontrados no headspace de carnes ovina e caprina cozidas, dentre os quais alguns são relatados na literatura como importantes marcadores de carne processada, como os compostos sulfurados dimetil sulfeto, dimetil dissulfeto, dimetil trissulfeto, 2-acetiltiazol e os aldeídos hexanal e nonanal. Ademais, essa diferenciação qualitativa dos compostos voláteis das carnes caprina e ovina, teve uma contribuição importante dos compostos terpênicos, sendo que vários deles foram encontrados exclusivamente em caprinos ( $\beta$-pineno, $\alpha$-gurjuneno, $\alpha$-muuroleno), podendo ser o indicativo de que esses animais, criados no mesmo pastejo, alimentam-se de vegetação diferenciada e/ou que seu metabolismo favorece a bioacumulação desses compostos nos tecidos avaliados. O extrato do headspace dos músculos V. lateralis e $\boldsymbol{L}$. dorsi da espécie ovina apresentou compostos voláteis que os discriminam e, possivelmente, podem estar envolvidos na diferenciação sensorial do aroma dessas carnes.

\section{AGRADECIMENTOS}

Este estudo foi financiado pelo Conselho Nacional de Desenvolvimento Científico e Tecnológico (CNPq) através de bolsas de produtividade em pesquisa e de iniciação científica $\mathrm{CNPq}$ / Programa Institucional de Bolsas de Iniciação Científica
(PIBIC), bem como pelo projeto Edital Universal, MCT/CNPq n. $14 / 2010$, processo 475903/2010-2

\section{REFERÊNCIAS}

ACREE, T.E.; HEINRICH, A. Flavornet and human odor space, gas chromatography - olfactometry (GC-O) of natural products, 10 jun. 2012. Disponível em: $<$ http://www.flavornet.org/f kovats. html $>$. Acesso em: 10 jun. 2012.

CHEN, W. et al. Anaerobic degradation of veratrylglycerol-bguaiacyl ether and guaiacoxyacetic acid by mixed rumen bacteria. Applied Environmental Microbiology, v.50, n.6, p.1451-1456, 1985.

CORNU, A. et al. Solid-phase microextraction of volatile components from natural grassland plants. Journal of Agricultural and Food Chemistry, v.49, n.1, p.203-209, 2001. Disponível em: <http://pubs.acs.org/doi/abs/10.1021/jf0008341>. Acesso em: 03 jun. 2012. doi: 10.1021/jf0008341.

DRANSFIELD, E. The taste of fat. Meat Science, v.80, n.1, p.37-42, 2008. Disponível em: <http://dx.doi.org/10.1016/j. meatsci.2008.05.030>. Acesso em: 11 set. 2012. doi: 10.1016/j. meatsci.2008.05.030.

ELMORE, J.S. et al. Two-fibre solid-phase microextraction combined with gas chromatography-mass spectrometry for the analysis of volatile aroma compounds in cooked pork. Journal of Chromatography A, v.905, p.233-240, 2000b. Disponível em: $<\mathrm{http}$ ://dx.doi.org/10.1016/S0021-9673(00)00990-0>. Acesso em: 05 jul. 2012.

ELMORE, J.S. et al. The effects of diet and breed on the volatile compounds of cooked lamb. Meat Science, v.55, n.2, p.149159, 2000a. Disponível em: <http://dx.doi.org/10.1016/S03091740(99)00137-0>. Acesso em: 07 jul. 2012. doi: 10.1016/S03091740(99)00137-0.

ELMORE, J.S. et al. Dietary manipulation of fatty acid composition in lamb meat and its effect on the volatile aroma compounds of grilled lambs. Meat Science, v.69, p.233-242, 2005. Disponível em: <http://dx.doi.org/10.1016/j.meatsci.2004.07.002>. Acesso em: 04 jul. 2012. doi: 10.1016/j.meatsci.2004.07.002.

EL-SAYED, A.M. The Pherobase, 10 jun. 2012. Disponível em: $<$ http://www.pherobase.com/database/kovats /kovats-index.php $>$. Acesso em: 10 de jun. 2012.

ESTÉVEZ, M. Protein carbonyls in meat systems: a review. Meat Science, v.89, p.259-279, 2011. Disponível em: $<$ http://dx.doi. org/10.1016/j.meatsci.2011.04.025>. Acesso em: 03 jun. 2012. doi:10.1016/j.meatsci.2011.04.025.

GARGOURI, M. et al. Synthesis of a novel macrolactone by lipase-catalyzed intra-esterification of hydroxy-fatty acid in organic media. Journal of Biotechnology, v.92, n.3, p.259266, 2003. Disponível em: <http://dx.doi.org/10.1016/S01681656(01)00374-1>. Acesso em: 10 set. 2012. doi: 10.1016/S01681656(01)00374-1.

HA, J.K.; LINDSAY, R.C. Volatiles alkylphenols and thiophenol in species-related characterising flavors of red meats. Journal of Food Science, v.56, p.1197-1202, 1991. Disponível em: <http:// onlinelibrary.wiley.com/doi/10.1111/j.1365-2621.1991.tb04733.x/ 
pdf $>$. Acesso em: 07 jun. 2012. doi: 10.1111/j.1365-2621.1991. tb04733.x.

MADRUGA, M.S. Studies on some factors affecting meat flavour formation. 1994. $412 \mathrm{p}$. $\mathrm{PhD}$ thesis - University of Reading, Reading, England, UK.

MADRUGA, M.S. Formação do aroma cárneo. Anais do Boletim da SBCTA, v.31, n.1, p.33-41, 1997.

MADRUGA, M.S. et al. Volatile flavour profile of goat meat extracted by three widely used techniques. Food Chemistry, v.115, p.1081-1087, 2009. Disponível em: <http://dx.doi. org/10.1016/j.foodchem.2008.12.065>. Acesso em: 07 jun. 2012. doi:10.1016/j.foodchem.2008.12.065

MARIACA, R.G. et al. Occurrence of volatile mono- and sesquiterpenoids in highland and lowland plant species as possible precursors for flavor compounds in milk and dairy products. Journal of Agricultural and Food Chemistry, v.45, p.44234434, 1997. Disponível em: <http://pubs.acs.org/doi/abs/10.1021/ jf970216t>. Acesso em: 11 jun. 2012. doi: 10.1021/jf970216t.

MOTTRAM, D.S. Flavour formation in meat and meat products: a review. Food Chemistry, v.62, n.4, p.415-424, 1998. doi:10.1016/j.meatsci.2011.04.025.

PRIOLO, A. et al. Fat volatiles tracers of grass feeding in sheep. Meat Science, v.66, n.2, p.475-481, 2004. doi: 10.1016/S03091740(03)00136-0.

SONDERGAARD, A.K.; STAHNKE, L.H. Growth and aroma production by Staphylococcus xylosus, S. carnosus and $S$. equorum - a comparative study in model systems. International Journal of Food Microbiology, v.75, p.99-109, 2002. Disponível em: <http://dx.doi.org/10.1016/S0168-1605(01)00729-2>. Acesso em: 02 jul. 2012. doi: 10.1016/S0168-1605(01)00729-2.

SUTHERLAND, M.M.; AMES. J.M. The effect of castration on the headspace aroma components of cooked lamb. Journal Science Food Agriculture, v.69, p.403-413, 1995. Disponível em: <http://onlinelibrary.wiley.com/doi/10.1002/jsfa.2740690402/ abstract>. Acesso em: 02 jul. 2012. doi: 10.1002/jsfa.2740690402.

URBACH, G. Effect of feed on flavor in dairy foods. Journal of Dairy Science, v.73, n.12, p.3639-3650, 1990. Disponível em: $<$ http://dx.doi.org/10.3168/jds.S0022-0302(90)79067-4>. Acesso em: 10 set. 2012. doi: 10.3168/jds.S0022-0302(90)79067-4.

VASTA, V., PRIOLO, A. Ruminant fat volatiles as affected by diet. A review. Meat Science, v.73, p.218-228, 2006. Disponível em: <http://dx.doi.org/10.1016/j.meatsci.2005.11.017>. Acesso em: 05 jul. 2012. doi: 10.1016/j.meatsci.2005.11.017.

VASTA, V. et al. The volatile profile of Longissimus dorsi muscle of heifers fed pasture, pasture silage or cereal concentrate: Implication for dietary discrimination, Meat Science, v.87, p.282-289, 2011. Disponível em: <http://dx.doi.org/10.1016/j. meatsci.2010.11.003>. Acesso em: 03 jul. 2012. doi: 10.1016/j. meatsci.2010.11.003.

YOUNG, O.A. et al. Pastoral and species flavour in lambs raised on pasture, lucerne or maize. Journal of the Science of Food and Agriculture, v.83, n.2, p.93-104, 2003. Disponível em: <http:// onlinelibrary.wiley.com/doi/10.1002/jsfa.1282/pdf>. Acesso em: 04 jul. 2012. doi: 10.1002/jsfa.1282. 